\title{
INTERACTION OF TACHYONS WITH MATTER
}

\author{
ROMAN TOMASCHITZ \\ Department of Physics, Hiroshima University, \\ 1-3-1 Kagami-yama, Higashi-Hiroshima 739-8526, Japan \\ E-mail:roman@fusion.sci.hiroshima-u.ac.jp
}

Received 7 July 1999

Revised 15 August 1999

\begin{abstract}
A new interaction mechanism of superluminal particles with matter is suggested. Tachyons are described by a real Proca field with negative mass square, coupled to a current of subluminal matter. The potential of a static point source in this field theory is a damped periodic function with $1 / r$-decay. We treat this potential as a perturbation of the Coulomb potential, and study its effects on cross-sections and energy levels. In the limit of large impact parameter, the periodicity of the potential has a pronounced effect on the classical cross-section, which gets singular at the accumulating extrema of the scattering angle. In this limit we define the cross-section wave mechanically, by semiclassical rainbow scattering. The impact of the tachyon potential on the energy levels of hydrogen and hydrogenic ions is calculated by means of Bohr-Sommerfeld quantization. Estimates for the tachyon mass $(3 \mathrm{keV})$ and the coupling constant of the tachyon potential are derived on the basis of high-precision Lamb shift measurements.
\end{abstract}

\section{Introduction}

Modern theories of superluminal motion ${ }^{1-6}$ are based on the formalism of classical relativistic mechanics. Faster-than-light particles (tachyons) are usually introduced as an extension of the relativistic particle concept, as particles with negative mass square, or, if one prefers, imaginary mass. ${ }^{7}$ In the relativistic Hamilton-Jacobi equation, this just means to assume $m^{2}<0$ without further alterations. If tachyons are supposed to carry electric charge, then their coupling to the electromagnetic potential is effected by minimal substitution, and the Lagrangian for tachyons can be written in the same way as for subluminal particles, $L=-\sqrt{-m^{2} \eta_{\mu \nu} \dot{x}^{\mu} \dot{x}^{\nu}}+e A_{\mu} \dot{x}^{\mu}$, but with $m^{2}<0$. $\left[\operatorname{diag}\left(\eta_{\mu \nu}\right)=(-1,1,1,1) ; \eta_{\mu \nu}\right.$ may be replaced by a Riemannian metric $g_{\mu \nu}$ of the same signature, of course.] Sommerfeld's pre-relativistic study of superluminal motion ${ }^{8}$ aimed at accelerating electrons beyond the speed of light by means of electromagnetic fields, but otherwise his view of tachyons as point particles coupled in the usual way to the electromagnetic field was taken over by modern authors.

In this paper, a different approach to superluminal motion is investigated. Superluminal wave propagation is modeled in analogy to classical electrodynamics, by 
a Proca equation with negative mass square, very contrary to the prevailing view of tachyons as charged point particles with imaginary mass and zero spin. The superluminal wave modes of the tachyon field are coupled to a current of subluminal massive particles by minimal substitution. In short, in the above Lagrangian we regard the field as superluminal rather than the particle, cf. the conclusion for further discussion on that. In the following we outline this field theory in a Robertson-Walker cosmology, and calculate the static potential, the analog to the Coulomb potential. The cosmic background is crucial in understanding the causality of superluminal signal transfer, as the expanding galaxy grid and the Planckian microwave radiation define a universal frame of reference and a unique cosmic time order for all uniformly moving observers. ${ }^{9-11}$ In this paper, however, we focus on local effects, on interactions in locally geodesic coordinates, and we will study the effects of the static potential of this field theory rather than signal transfer by tachyonic wave propagation.

We consider a Robertson-Walker cosmology with negatively curved three-space, with the line element $d s^{2}=-d \tau^{2}+a^{2}(\tau) d \sigma^{2}$. Because of the spherical symmetry of the static potential, we use as coordinate representation of the hyperbolic threespace the ball-model of hyperbolic space, $d \sigma^{2}=4\left(1-|\mathbf{x}|^{2}\right)^{-2} d \mathbf{x}^{2},|\mathbf{x}|<1$. The Lagrangian

$$
L=-\frac{1}{4} F_{\alpha \beta} F^{\alpha \beta}+\frac{1}{2} \mu^{2} A_{\alpha} A^{\alpha}+A_{\alpha} j^{\alpha},
$$

$F_{\alpha \beta}=A_{\beta, \alpha}-A_{\alpha, \beta}, \mu=m_{t} / a(\tau), m_{t}>0$, leads to a Proca equation ${ }^{12,13}$ with negative mass square $\left[\mu^{2}>0\right.$, in the notation of (1.1)],

$$
\frac{1}{\sqrt{-g}} \frac{\partial\left(\sqrt{-g} F^{\alpha \beta}\right)}{\partial x^{\beta}}-\mu^{2} A^{\alpha}=j^{\alpha},
$$

for a real vector potential $A_{\alpha}$. The tachyon mass $\mu$ is a scalar inversely proportional to the expansion factor, in order to retain the conformal coupling of the Maxwell field; also note that $\mu$ is not a rest mass, and Planck's constant enters into this classical field theory to give the mass term the right dimension in the Lagrangian, $\mu=m_{t} c /(\hbar a(\tau))$, with $a\left(\tau_{0}\right)=1$ at the present epoch. [The cosmic time scaling of the tachyon mass reminds us of the varying fundamental constants of Eddington, Milne, ${ }^{14}$ and Dirac, ${ }^{15} \mathrm{cf}$. Ref. 16. A time variation of the fundamental constants in an absolute (i.e. nonrelativistic) cosmic space-time conception (based on the microwave background), leading to a uniform contraction of measuring rods that manifests in dispersionless cosmic redshifts, has recently been proposed in Refs. 10, 17 and 18.] The Lagrangian (1.1) immediately suggests an interaction of the tachyon field with subluminal matter. The current in (1.2) is structured like in electrodynamics, generated by subluminal sources carrying tachyonic charge. In Ref. 19, we considered a cosmic tachyon background radiation based on the field equations (1.1). In this paper, we focus on the potential of a static point source; superluminal wave propagation in this field theory is studied in Ref. 20, also see the conclusion. 
The tachyon potential of a static point source is defined by the current $j^{0}=$ $q(-g)^{-1 / 2} \delta(\mathbf{x}), j^{k}=0$, in analogy to electrostatics; $q$ is the tachyon charge of this source, further discussed in Secs. 2 and 4. The potential is readily calculated from (1.2) as a linear combination of

$$
\begin{aligned}
& A_{0}=a^{-1} f_{ \pm}(r), \quad A_{i}=0, \\
& F_{0 i}=-\varphi(\tau) f_{ \pm}^{\prime}(r) x_{i} / r \\
& f_{ \pm}=r^{-1}\left(1-r^{2}\right)^{1 \mp i \sqrt{m_{t}^{2}-1}}(1-r)^{ \pm 2 i \sqrt{m_{t}^{2}-1}} .
\end{aligned}
$$

We assume $m_{t}^{2}>1$, cf. the discussion below. Thus the potential of a static point source reads as

$$
\begin{aligned}
A_{0} & =-\frac{q}{4 \pi a R} \frac{\cos (\kappa d(r))+\lambda \sin (\kappa d(r))}{\sinh \left(a^{-1} R^{-1} d(r)\right)}, \\
d(r) & :=R a(\tau) \log \frac{1+r / R}{1-r / R} \\
\kappa & :=\sqrt{\left(\frac{m_{t} c}{a \hbar}\right)^{2}-\frac{1}{(R a)^{2}}}
\end{aligned}
$$

we have restored here the natural units; $d(r)$ is the radial distance function of the cosmic 3-space [curvature radius $R a(\tau)$ ], and $\lambda$ is an integration parameter. Both fundamental solutions (1.3) have the same exponential decay at infinity, i.e. for distances larger than the curvature radius; so one cannot select a special solution on the grounds of fastest decay, which is the usual argument to fix the potential in a field theory with nonnegative mass square. However, we can require the tachyon potential (1.4) to be singularity free at $r=0$, so that it has a finite classical selfenergy, ${ }^{21}$ which means to drop the cosine and to put $\lambda=1$. This seems to me the natural choice, but in the following we will not specify a boundary condition, apart from Sec. 4, where we connect to Lamb shift measurements on the basis of a pure sine potential.

We will focus on the local Euclidean limit of the potential (1.4), which is readily obtained by identifying $d(r)$ with the Euclidean distance and by performing the limit $R \rightarrow \infty$, cf. (2.1). There is of course no periodicity in the potential (1.4) if $\kappa$ is imaginary or zero, but this requires in the limit $R \rightarrow \infty$ a nonnegative mass square (i.e. $m_{t}^{2} \leq 0$ in our notation).

The purpose of this paper is to point out possible manifestations of the tachyon potential in atomic cross-sections and bound states. In Sec. 2, we study classical scattering in the Euclidean limit of the potential (1.4) superposed on the Coulomb potential. We calculate the correction to the Rutherford cross-section generated by the tachyon potential, and compare it to the relativistic correction. If the impact parameter is large, the deflection angle is dominated by the tachyon rather than 
the Coulomb potential. As the tachyon potential is a damped periodic function, the scattering angle has accumulating extrema in this limit; the classical cross-section becomes ill-defined, and one has to resort to semiclassical mechanics. In Sec. 3, we study the quantum mechanical cross-section in the WKB limit of large angular momentum and impact parameter. Borrowing from rainbow scattering, ${ }^{22-24}$ we calculate the cross-section at the accumulating extrema of the scattering angle.

In Sec. 4, we study the effect of the tachyon potential on energy levels by BohrSommerfeld quantization. We derive a lower bound of $3 \mathrm{keV}$ for the tachyon mass. This is done by comparing high-precision measurements of the $1 S-2 S_{1 / 2}$ and Ly- $\alpha_{1}$ transitions in hydrogen with Lamb shift calculations. An estimate for the coupling constant of the tachyon potential is likewise obtained in this way. Finally we study frequency shifts induced by the tachyon potential in medium and high- $Z$ hydrogenlike ions. We obtain the $Z$-scaling of the tachyonic coupling constant and relate tachyonic to electric charge. In Sec. 5 we present our conclusions and give estimates on tachyonic black-body radiation and on atomic absorption and emission rates for tachyon radiation.

\section{Scattering Effects of the Tachyon Potential}

We consider the local Euclidean limit of the tachyon potential (1.6), and regard this potential as a perturbation of the Coulomb potential,

$$
U(r)=\frac{\alpha}{r}+\frac{\beta}{r}(\cos (\kappa r)+\lambda \sin (\kappa r)),
$$

$\kappa:=m_{t} c / \hbar>0$. We assume $|\beta / \alpha| \ll 1$, and $|\lambda \beta / \alpha| \ll 1$, so that we can treat the $\beta$ term (denoted by $U_{\beta}$ in the following) perturbatively. We put $\alpha=e_{1} e_{2} /(4 \pi)$, and $\beta=-q_{1} q_{2} /(4 \pi)$, where $e_{1,2}$ and $q_{1,2}$ are the electric and tachyonic charges of source and particle. The tachyon potential clearly reminds us of the "fifth force" 25 added to the gravitational potential, so that it is tempting to relate the tachyon charge $q$ to $e$; this will be done at the end of Sec. 4 . A sine potential is obtained by substituting $\beta / \lambda$ for $\beta$, and by performing the limit $\lambda \rightarrow \infty$; this procedure can easily be carried out in all the following results for cross-sections and energy levels. As already emphasized in the introduction, we do not study superluminal signals in this paper (see, however, the end of Sec. 5). The sources carrying tachyonic and electric charge are always subluminal, and the tachyon potential is not related to tachyonic wave propagation, namely to wave solutions of Eq. (1.2), which are discussed in Ref. 20. Nevertheless, the perturbation (2.1) of the Coulomb potential gives indirect evidence for superluminal signals, in the same way as the Coulomb potential is an indication for electromagnetic waves, when considered as a solution of Maxwell's equations. Tachyons, superluminal particles, emerge via the geometric optics limit of the field equations (1.2). This is shortly discussed in the conclusion, otherwise we study throughout this paper subluminal particle motion in the static potential (2.1). 
In this section, we investigate classical scattering in the potential (2.1). The scattered particles carry electric and tachyonic charge and are, as mentioned, subluminal. The Hamilton-Jacobi equation reads

$$
-c^{-2}\left(\frac{\partial S}{\partial t}+U(r)\right)^{2}+\left(\frac{\partial S}{\partial r}\right)^{2}+r^{-2}\left(\frac{\partial S}{\partial \varphi}\right)^{2}=-m^{2} c^{2}
$$

$m$ denotes the mass of the scattered subluminal particle and should not be confused with the tachyon mass $m_{t}$ in the Proca equation, which enters via $\kappa$ in the potential (2.1). Equation (2.2) is solved by the ansatz $S=-\left(E+m c^{2}\right) t+M \varphi+S(r)$,

$$
\begin{aligned}
S(R) & :=\int_{r_{\min }}^{R}\left[2 m(E-U)-M^{2} r^{-2}+c^{-2}(E-U)^{2}\right]^{1 / 2} d r \\
& =\int_{r_{\min }}^{R}\left[p_{c}^{2}-M^{2} r^{-2}-2 c^{-2} E_{c} U(r)+c^{-2} U^{2}(r)\right]^{1 / 2} d r,
\end{aligned}
$$

where we introduced $E_{c}=E+m c^{2}=m c^{2}\left(1-v^{2} / c^{2}\right)^{-1 / 2}$, and $p_{c}=E_{c} v / c^{2}$. $(R$ denotes the radial coordinate and should not be mixed up with the curvature radius of the cosmic three-space defined in Sec. 1.) $v$ is the absolute value of the asymptotic velocity, and $r_{\min }$ is the positive root of the integrand.

First we study two simple limit cases. By expanding the integrand linearly in $\alpha$ and $\beta$, we arrive at

$$
S(R)=\int_{M / p_{c}}^{R} \sqrt{p_{c}^{2}-M^{2} r^{-2}} d r-\frac{E_{c}}{c^{2}} \int_{M / p_{c}}^{R} \frac{U(r) d r}{\sqrt{p_{c}^{2}-M^{2} r^{-2}}} .
$$

To calculate the deflection angle, $\varphi=-\partial S / \partial M, R \rightarrow \infty$, we have to evaluate (2.5) for large $R$. Performing the integrations, we obtain

$$
S(R) \approx p_{c} R-\frac{\pi}{2} M+\alpha \frac{E_{c}}{c^{2} p_{c}} \log \frac{M}{2 p_{c} R}+\beta \frac{E_{c}}{c^{2} p_{c}} \frac{\pi}{2}\left(N_{0}\left(\frac{\kappa M}{p_{c}}\right)-\lambda J_{0}\left(\frac{\kappa M}{p_{c}}\right)\right),
$$

where we have dropped terms vanishing for $R \rightarrow \infty$. $J_{0}$ and $N_{0}$ are the usual Bessel and Neumann functions. ${ }^{26,27}$ (The integral over the $\beta$ term of the potential is finite and standard for $R=\infty$, cf. Ref. 26.) We find the deflection angle

$$
\varphi(\rho)=\frac{\pi}{2}-\frac{\alpha E_{c}}{c^{2} p_{c}^{2}} \frac{1}{\rho}+\beta \kappa \frac{E_{c}}{c^{2} p_{c}^{2}} \frac{\pi}{2}\left(N_{1}(\kappa \rho)-\lambda J_{1}(\kappa \rho)\right),
$$

where we have expressed the angular momentum by the impact parameter $\rho=$ $M / p_{c}$. Evidently, this expansion is not valid in the limit $\rho \rightarrow 0$, as the coefficients diverge.

Next, we calculate the deflection angle in the limit of large angular momentum, $M \rightarrow \infty$. We insert a convergence factor $r^{-\delta}$ into (2.4), and put $R=\infty$, arriving in this way at

$$
S=M^{1-\delta} \int_{r_{\min } / M}^{\infty} r^{-\delta}\left[p_{c}^{2}-r^{-2}-2 c^{-2} E_{c} U(M r)+c^{-2} U^{2}(M r)\right]^{1 / 2} d r
$$


$r_{\min } / M$ is the positive root of the radical. Expanding in powers of $1 / M$, we obtain

$$
\begin{aligned}
S \approx & M \int_{1 / p_{c}}^{\infty} d r\left(r^{-\delta} \sqrt{p_{c}^{2}-r^{-2}}-\frac{\alpha E_{c}}{c^{2}} \frac{1}{r^{\delta} \sqrt{p_{c}^{2} r^{2}-1}}\right. \\
& \left.-\frac{\beta E_{c}}{c^{2}} \frac{\cos \left(r_{\min } p_{c} \kappa r\right)+\lambda \sin \left(r_{\min } p_{c} \kappa r\right)}{\sqrt{p_{c}^{2} r^{2}-1}}\right) .
\end{aligned}
$$

We have here already dropped the convergence factor (i.e. put $\delta=0$ ) wherever possible. The first two terms in (2.9) give $\Gamma$ functions, and the trigonometric integral has the same structure as in (2.5). The deflection angle, $\varphi=-\partial S / \partial M, \delta \rightarrow 0$, reads

$$
\begin{aligned}
\varphi(\rho) & =\frac{\pi}{2}+\frac{\beta E_{c}}{c^{2} p_{c}^{2}} \sqrt{\frac{\pi \kappa}{2}} \frac{1}{\rho^{1 / 2}}(\sin \vartheta-\lambda \cos \vartheta+O(\beta))-\frac{\alpha E_{c}}{c^{2} p_{c}^{2}} \frac{1}{\rho}+O\left(\frac{1}{\rho^{3 / 2}}\right), \\
\vartheta & :=\kappa \rho+\frac{\kappa \alpha E_{c}}{c^{2} p_{c}^{2}}-\frac{3}{4} \pi .
\end{aligned}
$$

This result coincides with (2.7), provided we use the asymptotic expressions $\left(J_{1}, N_{1}\right)(z) \sim \sqrt{2 /(\pi z)}(\cos , \sin )(z-3 \pi / 4)$ there and drop the $\alpha$ term in the trigonometric functions in (2.10). The expansion (2.7) is linear in $\alpha$ and $\beta$, whereas Eq. (2.10) is linear in $\beta$ and only valid for large impact parameter, but it takes the Coulomb potential fully into account. The $\beta$ term in (2.10) dominates the contribution of the Coulomb potential if the impact parameter is sufficiently large; $\varphi(\rho)$ becomes a damped harmonic function, and the Coulomb potential enters as a phase shift.

Finally we calculate the deflection angle without assuming a large impact parameter, and treat the Coulomb potential nonperturbatively. We consider corrections linear in $\beta$ and $1 / c^{2}$; terms of the order $\beta / c^{2}$ are dropped. We may thus approximate (2.3) by

$$
S(R)=\int_{r_{\min }}^{R}\left[2 m\left(\tilde{E}-\frac{\tilde{\alpha}}{r}\right)-\frac{M^{2}}{r^{2}}+\frac{1}{c^{2}} \frac{\alpha^{2}}{r^{2}}-2 m U_{\beta}(r)\right]^{1 / 2} d r,
$$

with $\tilde{E}:=E\left(1+E /\left(2 m c^{2}\right)\right), \tilde{\alpha}:=\alpha\left(1+E /\left(m c^{2}\right)\right)$, and $E=m v^{2} / 2 . r_{\text {min }}$ is the positive root of the radical. We expand $S(R)$ in $\beta$ and $1 / c^{2}, S=S_{0}+S^{\text {rel }}+S^{\text {tach }}$,

$$
\begin{aligned}
S_{0} & =\int_{\tilde{r}_{\text {min }}}^{R}\left[2 m\left(\tilde{E}-\tilde{\alpha} r^{-1}\right)-M^{2} r^{-2}\right]^{1 / 2} d r \\
S^{\text {rel }} & =\frac{1}{2} \frac{\alpha^{2}}{c^{2}} \int_{r_{\text {min }}}^{\infty} \frac{1}{\left[2 m\left(E-\alpha r^{-1}\right)-M^{2} r^{-2}\right]^{1 / 2}} \frac{1}{r^{2}} d r, \\
S^{\text {tach }} & =-m \beta \int_{r_{\min }}^{\infty} \frac{\cos (\kappa r)+\lambda \sin (\kappa r)}{\left[2 m\left(E-\alpha r^{-1}\right)-M^{2} r^{-2}\right]^{1 / 2}} \frac{1}{r} d r .
\end{aligned}
$$


In (2.12) and (2.13) we have put $R=\infty$, as these integrals are already convergent, and we are interested only in terms which give a nonvanishing contribution for $R \rightarrow \infty \cdot r_{\min }$ and $\tilde{r}_{\min }$ denote the positive roots of the radicals in the respective integrands. [Note that $r_{\min }$ as defined by the radical in (2.11) differs from the root of the radical in (2.13) and (2.14) by terms of order $O\left(\beta, c^{-2}\right)$; the justification for that is given in the Remark following (4.6).] We obtain, by elementary integration,

$$
\begin{aligned}
S_{0}= & \sqrt{2 m \tilde{E}} R-\sqrt{\frac{m}{2 \tilde{E}}} \tilde{\alpha}[1+\log (2 R)]-\frac{\pi}{2} M \\
& +M \arcsin \left(\frac{\tilde{\alpha}}{2 \tilde{E}}\left(\frac{\tilde{\alpha}^{2}}{4 \tilde{E}^{2}}+\frac{M^{2}}{2 m \tilde{E}}\right)^{-1 / 2}\right) \\
& +\sqrt{\frac{m}{2 \tilde{E}}} \tilde{\alpha} \log \sqrt{\frac{\tilde{\alpha}^{2}}{4 \tilde{E}^{2}}+\frac{M^{2}}{2 m \tilde{E}}}+O\left(\frac{1}{R}\right), \\
S^{\mathrm{rel}}= & \frac{1}{2} \frac{\alpha^{2}}{c^{2}} \frac{1}{M}\left[\frac{\pi}{2}-\arcsin \left(\frac{\alpha}{2 E}\left(\frac{\alpha^{2}}{4 E^{2}}+\frac{M^{2}}{2 m E}\right)^{-1 / 2}\right)\right],
\end{aligned}
$$

with $-\pi / 2 \leq \arcsin \leq \pi / 2 . \tilde{E}$ and $\tilde{\alpha}$ are defined after (2.11). As for the calculation of $S^{\text {tach }}$, it is convenient to introduce as parameters the semiaxis and the eccentricity of the hyperbola of nonrelativistic motion in the Coulomb potential, $a=|\alpha| /(2 E)$ and $e=\sqrt{1+2 E M^{2} /\left(\alpha^{2} m\right)}$. The two roots of the radical in (2.13) read $r_{\min }=$ $a(\operatorname{sign}(\alpha)+e)$ and $\hat{r}=a(\operatorname{sign}(\alpha)-e)$ and we find

$$
\begin{aligned}
S^{\text {tach }} & =-\beta \sqrt{\frac{m}{2 E}} \int_{r_{\min }}^{\infty} \frac{\cos (\kappa r)+\lambda \sin (\kappa r)}{\sqrt{\left(r-r_{\min }\right)(r-\hat{r})}} d r \\
& =\frac{\beta}{v} \frac{\pi}{2}\left[A J_{0}(\kappa a e)+B N_{0}(\kappa a e)\right], \\
A & :=\sin \left(\frac{\kappa \alpha}{m v^{2}}\right)-\lambda \cos \left(\frac{\kappa \alpha}{m v^{2}}\right), \\
B & =\cos \left(\frac{\kappa \alpha}{m v^{2}}\right)+\lambda \sin \left(\frac{\kappa \alpha}{m v^{2}}\right),
\end{aligned}
$$

where we have put $E=m v^{2} / 2$ (asymptotic velocity).

The contribution of $S_{0}$ to the deflection angle reads

$$
-\frac{\partial S_{0}}{\partial M}=\frac{\pi}{2}-\arcsin \frac{\alpha}{\sqrt{\alpha^{2}+m^{2} v^{4} \rho^{2}}}+\frac{1}{2 c^{2}} \frac{\alpha \rho m v^{4}}{\alpha^{2}+m^{2} v^{4} \rho^{2}} .
$$


[We have inserted here $M=\rho p_{c}$ as in (2.7), and then expanded in $1 / c^{2}$.] Analogously, we find, via (2.16) and (2.17),

$$
\begin{aligned}
-\frac{\partial S_{\mathrm{rel}}}{\partial M}= & \frac{1}{2 c^{2}} \frac{\alpha^{2}}{m^{2} v^{2} \rho^{2}}\left(\frac{\pi}{2}-\arcsin \frac{\alpha}{\sqrt{\alpha^{2}+m^{2} v^{4} \rho^{2}}}\right) \\
& -\frac{1}{2 m c^{2} \rho} \frac{\alpha^{3}}{\alpha^{2}+m^{2} v^{4} \rho^{2}}, \\
-\frac{\partial S_{\mathrm{tach}}}{\partial M}= & \frac{\pi}{2} \frac{\beta \kappa \rho}{\sqrt{\alpha^{2}+m^{2} v^{4} \rho^{2}}}\left(A J_{1}\left(\frac{\kappa}{m v^{2}} \sqrt{\alpha^{2}+m^{2} v^{4} \rho^{2}}\right)\right. \\
& \left.+B N_{1}\left(\frac{\kappa}{m v^{2}} \sqrt{\alpha^{2}+m^{2} v^{4} \rho^{2}}\right)\right)=: \beta T(\alpha, \rho) .
\end{aligned}
$$

The coefficients $A$ and $B$ in (2.20) are defined in (2.17). [We used $\partial e / \partial M=$ $\left(e^{2}-1\right) /(e M)$, and $J_{0}^{\prime}=-J_{1}, N_{0}^{\prime}=-N_{1}$ in (2.17).] In this way we find for the deflection angle

$$
\varphi(\rho)=\frac{\pi}{2}(1-\operatorname{sign}(\alpha))+\arctan \frac{m v^{2} \rho}{\alpha}+\frac{1}{c^{2}} R(\rho)+\beta T(\alpha, \rho),
$$

$(-\pi / 2 \leq \arctan \leq \pi / 2)$, with $\beta T(\alpha, \rho)$ as in $(2.20)$ and

$$
R(\rho):=\frac{\alpha^{2}}{2 m^{2} v^{2} \rho^{2}}\left(\frac{\pi}{2}(1-\operatorname{sign}(\alpha))+\arctan \frac{m v^{2} \rho}{\alpha}\right)+\frac{\alpha}{2 m \rho} \frac{m^{2} v^{4} \rho^{2}-\alpha^{2}}{m^{2} v^{4} \rho^{2}+\alpha^{2}} .
$$

In the case of a repulsive Coulomb potential, $\alpha>0$, we have $0<\varphi(\rho)<\pi / 2$, and if $\alpha<0$, then $\pi / 2<\varphi(\rho)<\pi$. If we expand (2.21) in $\alpha$ and $(2.7)$ in $1 / c^{2}$, then these limits coincide, of course. The asymptotic limit $\rho \rightarrow \infty$ of Eq. (2.21) coincides with (2.10), if we expand (2.10) in $1 / c^{2}$. The limit $\rho \rightarrow 0$ in (2.21) can only be performed if $\alpha>0$; in an attractive Coulomb potential, $\alpha<0$, the expansion (2.21) breaks down in this limit, because of the relativistic term, which diverges as $R(\rho) \sim 1 / \rho^{2}$. This is a well-known relativistic effect; the trajectory is not any more close to hyperbolic, but spirals into the scatterer. ${ }^{23}$

To calculate the classical cross-section, we have to express the impact parameter as a function of the deflection angle. If $\alpha>0$, then $0<\varphi<\pi / 2$, and the scattering angle reads $\chi=\pi-2 \varphi$. If $\alpha<0$, we have $\pi / 2<\varphi<\pi$, and $\chi=2 \varphi-\pi$. In either case, $0<\chi<\pi$. We readily calculate, by inverting (2.21),

$$
\begin{aligned}
& \rho(\chi)=\frac{|\alpha|}{m v^{2}} \cot (\chi / 2)\left[1-\frac{v^{2}}{c^{2}} h(\chi)-\frac{\beta}{\alpha} g(\chi)\right], \\
& h(\chi):=\frac{1}{2}\left(1-\tan ^{2}(\chi / 2)+\left(\operatorname{sign}(\alpha) \frac{\pi}{2}-\frac{\chi}{2}\right) \frac{\tan (\chi / 2)}{\cos ^{2}(\chi / 2)}\right), \\
& g(\chi):=\frac{\pi}{2} \xi\left(A J_{1}(\xi)+B N_{1}(\xi)\right), \quad \xi:=\frac{|\alpha| \kappa}{m v^{2}} \frac{1}{\sin (\chi / 2)} .
\end{aligned}
$$


The classical cross-section, $d \sigma=2 \pi \rho|d \rho / d \chi| d \chi$, reads as

$$
\begin{aligned}
d \sigma & =\pi \frac{\alpha^{2}}{m^{2} v^{4}} \frac{\cot (\chi / 2)}{\sin ^{2}(\chi / 2)} d \chi\left(1-\frac{v^{2}}{c^{2}} H_{\sigma}(\chi)-\frac{\beta}{\alpha} G_{\sigma}(\chi)\right) \\
H_{\sigma}(\chi) & =1+\frac{1}{2} \tan ^{2}(\chi / 2)-\left(\operatorname{sign}(\alpha) \frac{\pi}{2}-\frac{\chi}{2}\right) \tan (\chi / 2)\left(\frac{1}{2 \cos ^{2}(\chi / 2)}-1\right), \\
G_{\sigma}(\chi) & =\pi \xi\left(A J_{1}(\xi)+B N_{1}(\xi)\right)+\frac{\pi}{2} \cos ^{2}\left(\frac{\chi}{2}\right) \xi^{2}\left(A J_{0}(\xi)+B N_{0}(\xi)\right)
\end{aligned}
$$

with $\xi$ as in (2.23) and $A, B$ as in (2.17).

In the limit $\chi \rightarrow 0$ [i.e. $\rho \rightarrow \infty$, cf. (2.10)], the expansions (2.23) and (2.24) break down, because $g(\chi) \sim \chi^{-1 / 2}$ and $G_{\sigma}(\chi) \sim \chi^{-3 / 2}$. In this limit we calculate the cross-section quasiclassically in Sec. 3. We may use (2.24) for small $\chi$ as long as $\xi^{3 / 2}(\chi) \ll|\alpha / \beta|$ holds. In the limit $\xi \rightarrow 0$, we find $G_{\sigma}(\chi) \sim 2 g(\chi)$ and $1+g(\chi) \sim-\lambda \alpha \kappa /\left(m v^{2}\right)$. If the Coulomb potential is treated perturbatively in linear order [i.e. if we consider only terms linear in $\alpha, \beta$, and $1 / c^{2}$, cf. (2.7)], then $g(\chi) \sim-1$, and we obtain the Rutherford cross-section for a charge $\alpha+\beta$. This limit is to be compared with the quantum mechanical cross-section, $d \sigma=4 \pi \sin (\chi / 2) \cos (\chi / 2)|f(\chi)|^{2} d \chi$, with the scattering amplitude in Born approximation given by

$$
f(\chi)=-\frac{2 m}{\hbar^{2} q} \int_{0}^{\infty} e^{-\epsilon r} U(r) \sin (q r) d r=-\frac{2 m}{\hbar^{2} q^{2}}\left(\alpha+\beta \frac{q^{2}}{q^{2}-\kappa^{2}}\right)
$$

where $\epsilon \rightarrow 0, q=2 k \sin (\chi / 2), k=m v / \hbar$, and $U(r)$ as in (2.1). There is a singularity at $q=\kappa$. The Born approximation applies for large $k$, and this singularity just means that it breaks down for small $\chi$ (i.e. large impact parameter), when the tachyon potential dominates the contribution of the Coulomb potential in the classical cross-section [cf. (2.10)]. In the limit $\chi \rightarrow 0$ large phase shifts emerge, and WKB scattering applies instead [cf. Sec. 3]. For $\kappa^{2} / q^{2} \ll 1$ (large momentum and moderate scattering angle), the Born approximation is reliable, and we obtain, with $|\beta / \alpha| \ll 1$ (assumed throughout this paper),

$$
d \sigma=\frac{\pi(\alpha+\beta)^{2}}{m^{2} v^{4}} \frac{\cot (\chi / 2)}{\sin ^{2}(\chi / 2)} d \chi\left(1+\frac{1}{2} \frac{\beta}{\alpha} \frac{\kappa^{2} \hbar^{2}}{m^{2} v^{2}} \frac{1}{\sin ^{2}(\chi / 2)}\right) .
$$

Note that the $\lambda$ term of the tachyon potential $U_{\beta}(r)$ in (2.1) does not appear in this approximation. This is not surprising, since the Coulomb potential $\alpha / r$ is treated as perturbation in linear order in (2.25), and in the classical result (2.24), terms linear in $\alpha$ are likewise independent of $\lambda$, see the discussion following (2.24). The quantum mechanical analogue to the cross-section (2.24) is a first-order Born approximation for $U_{\beta}(r)$ based on the exact eigenfunctions of the Coulomb problem. 


\section{The Semiclassical Rainbow Limit}

We study scattering with large impact parameter in the potential (2.1). In this limit we have to use the quantum mechanical definition of the cross-section, as the classical cross-section gets singular at the accumulating extrema of the deflection angle [cf. (2.10)]. We focus in this section on nonrelativistic semiclassical scattering, with the Hamilton-Jacobi equation (2.2) being replaced by a Schrödinger equation with potential (2.1). The techniques employed are familiar from rainbow scattering, ${ }^{22,23}$ which justifies brevity. The scattering amplitude may be written as

$$
f(\chi)=\frac{1}{i k} \sum_{l=0}^{\infty}\left(l+\frac{1}{2}\right) P_{l}(\cos \chi) \exp \left(2 \delta_{l}\right) .
$$

Large impact parameter can only be realized by large angular momentum, as long as we consider nonrelativistic velocities, so we may apply WKB asymptotics to evaluate (3.1). The semiclassical phase shifts satisfy ${ }^{24}$

$$
\frac{d \delta_{l}}{d l}=\frac{\pi}{2}+\frac{1}{\hbar} \frac{\partial}{\partial l} \int_{r_{\min }}^{R \rightarrow \infty}\left[2 m(E-U)-\hbar^{2}\left(l+\frac{1}{2}\right)^{2} r^{-2}\right]^{1 / 2} d r
$$

$r_{\min }$ is the positive root of the radical. If we identify $m v=\hbar k, M=\hbar(l+1 / 2)$, and $\rho=(l+1 / 2) / k$, we find

$$
\begin{aligned}
\frac{d \delta_{l}}{d l} & \sim \frac{\pi}{2}-\varphi(\rho) \sim-A(\rho), \\
A(\rho) & =\frac{\beta}{m v^{2}} \sqrt{\frac{\pi \kappa}{2 \rho}}(\sin \xi-\lambda \cos \xi), \quad \xi:=\kappa \rho+\frac{\alpha \kappa}{m v^{2}}-\frac{3}{4} \pi,
\end{aligned}
$$

with $\varphi(\rho)$ as in (2.10) (and relativistic corrections dropped). Since scattering and deflection angle are related by $\chi=|2 \varphi-\pi|$, we have

$$
\chi=2|A(\rho)|, \quad 2 \frac{d \delta_{l}}{d l}+\operatorname{sign}(A(\rho)) \chi=0 .
$$

As the scattering amplitude (3.1) is dominated by large $l$-values, we may replace the Legendre polynomials by

$$
P_{l}(\cos \chi) \sim-(2 \pi l \sin \chi)^{-1 / 2}[i \exp (i(l+1 / 2) \chi+i \pi / 4)+\text { c.c. }] .
$$

The series (3.1) with (3.6) substituted is dominated by the stationary points of the phases, determined by $2 d \delta_{l} / d l \pm \chi=0$. We may therefore write for the scattering amplitude

$$
f(\chi) \sim-\frac{\operatorname{sign}(A(\rho))}{k \sqrt{2 \pi \sin \chi}} \sum_{l} l^{1 / 2} \exp i\left[2 \delta_{l}+\operatorname{sign}(A)\left(\left(l+\frac{1}{2}\right) \chi+\frac{\pi}{4}\right)\right] .
$$

Next we evaluate this series at an extremal scattering angle, $d \chi / d \rho=0$, and denote this extremum by $\left(\chi_{n}, \rho_{n}\right), \rho_{n}=\left(l_{n}+1 / 2\right) / k$. We expand $\delta_{l}$ by means of $(3.5)$,

$$
2 \delta_{l}=2 \delta_{l_{n}}-\operatorname{sign}\left(A\left(\rho_{n}\right)\right) \chi_{n}\left(l-l_{n}\right)+\left(\frac{\delta_{l_{n}}^{(3)}}{3}\right)\left(l-l_{n}\right)^{3}+\cdots,
$$


insert this expression into (3.7), replace the $l$ summation by an integral, and evaluate this by way of steepest descent,

$$
\begin{aligned}
\left|f\left(\chi_{n}\right)\right| \sqrt{2 \pi \sin \chi_{n}} & \sim \frac{l_{n}^{1 / 2}}{k} \int_{-\infty}^{\infty} \exp i\left(\frac{1}{3} \delta_{l_{n}}^{(3)}\left(l-l_{n}\right)^{3}\right) d l \\
& =\frac{l_{n}^{1 / 2}}{k} \frac{\Gamma(1 / 3)}{3^{1 / 6}}\left|\delta_{l_{n}}^{(3)}\right|^{-1 / 3} .
\end{aligned}
$$

(At an extremal scattering angle, there is no linear term in the exponent, and so Airy's integral reduces to a $\Gamma$ function.) What remains is to determine the extrema $\left(\chi_{n}, \rho_{n}\right)$ of $\chi(\rho)$, and to express in (3.9) $l_{n}$ and $\left|\delta_{l_{n}}^{(3)}\right|$ as functions of $\chi_{n}$. We have $\left|\delta_{l_{n}}^{(3)}\right|=\left|A^{\prime \prime}\left(\rho_{n}\right)\right|$, and the extrema of $\chi(\rho)$ coincide with those of $A(\rho)$ [cf. (3.5)]. We easily find, for $n \rightarrow \infty$,

$$
\begin{array}{rlrl}
\rho_{n} & \sim \frac{n \pi}{\kappa}, \quad \chi_{n}=2\left|A\left(\rho_{n}\right)\right| \sim \frac{|\beta| \kappa \sqrt{2\left(1+\lambda^{2}\right)}}{m v^{2}} \frac{1}{\sqrt{n}}, \\
\left|\delta_{l_{n}}^{(3)}\right| & \sim \frac{\kappa^{2}}{k^{2}}\left|A\left(\rho_{n}\right)\right|, \quad & l_{n} & \sim \frac{2 \pi \beta^{2} \kappa k\left(1+\lambda^{2}\right)}{m^{2} v^{4}} \frac{1}{\chi_{n}^{2}} .
\end{array}
$$

The differential cross-section, $d \sigma=2 \pi|f(\chi)|^{2} \sin \chi d \chi$, follows from (3.11) and (3.9) as

$$
d \sigma=\frac{2^{5 / 3}}{3^{1 / 3}} \pi \Gamma^{2}(1 / 3) \frac{\beta^{2}\left(1+\lambda^{2}\right) d \chi}{\left(\hbar \kappa m^{5} v^{11} \chi^{8}\right)^{1 / 3}} .
$$

We have here substituted $k=m v / \hbar$, and replaced $\chi_{n}$ by the continuous variable $\chi$, since $\chi_{n}-\chi_{n+1} \sim n^{-3 / 2}$. There is no classical limit of (3.12), as the classical cross-section cannot be defined at the extrema of the scattering angle.

Let us finally consider scattering in a regime $\left(\rho_{0}, \chi_{0}\right)$, where the impact parameter is still large enough for semiclassical scattering to apply, but so that the Coulomb term in (2.11) stays dominant, cf. the condition on $\chi$ given after (2.24). We may then expand $\delta_{l}$ as

$$
2 \delta_{l}=2 \delta_{l_{0}}-\operatorname{sign}\left(A\left(\rho_{0}\right)\right) \chi_{0}\left(l-l_{0}\right)+\delta_{l_{0}}^{(2)}\left(l-l_{0}\right)^{2}+\cdots,
$$

with $\delta_{l_{0}}^{(2)}=-k^{-1} A^{\prime}\left(\rho_{0}\right)$. $A(\rho)$ is defined by $(3.3)$ with $\varphi(\rho)$ as in (2.21). If we insert (3.13) into (3.7), replace the summation by an integral, and evaluate the stationary phase, we obtain instead of (3.9)

$$
\begin{aligned}
\left|f\left(\chi_{0}\right)\right| \sqrt{2 \pi \sin \chi_{0}} & \sim \frac{l_{0}^{1 / 2}}{k} \int_{-\infty}^{\infty} \exp \left(-\frac{1}{k}\left|A^{\prime}\left(\rho_{0}\right)\right|\left(l-l_{0}\right)^{2}\right) d l \\
& =\sqrt{\frac{\pi l_{0}}{k\left|A^{\prime}\left(\rho_{0}\right)\right|}} .
\end{aligned}
$$

Therefore, writing $\chi$ and $l$ for $\chi_{0}$ and $l_{0}$, we find via (3.3) $d \sigma \sim 2 \pi l k^{-1}|d \rho / d \chi| d \chi$, the classical cross-section. 


\section{The Effect of the Tachyon Potential on Bound States in Hydrogen and Hydrogenic Ions}

In this section we study bound states in the perturbed Coulomb potential (2.1), with $\alpha<0$; in particular we calculate level shifts induced by the tachyon potential in hydrogen and hydrogenlike ions. By assuming that these shifts can account for the discrepancy of experimental and theoretical Lamb shifts, we obtain estimates for the tachyon mass $m_{t}$ and the coupling constant $\beta$ of the tachyon potential, cf. (2.1). The action reads as in (2.3), but with $E<0$, so that there is a second positive root $r_{\max }$ of the integrand. To apply Bohr quantization, we have to evaluate the action integral

$$
S\left(r_{\min }, r_{\max }\right)=\int_{r_{\min }}^{r_{\max }}\left[2 m\left(-|\tilde{E}|+\frac{|\tilde{\alpha}|}{r}\right)-M^{2} r^{-2}+\frac{1}{c^{2}} \frac{|\alpha|^{2}}{r^{2}}-2 m U_{\beta}(r)\right]^{1 / 2} d r,
$$

with $\tilde{E}$ and $\tilde{\alpha}$ as defined after (2.11) (with negative $E$ and $\alpha$ ). $r_{\min } / \max$ are the roots of the integrand. Expanding in $\beta$ and $1 / c^{2}$, we obtain in linear order

$$
\begin{aligned}
S= & \int_{\tilde{r}_{\min }}^{\tilde{r}_{\max }}\left[2 m\left(-|\tilde{E}|+|\tilde{\alpha}| r^{-1}\right)-M^{2} r^{-2}\right]^{1 / 2} d r \\
& +\frac{1}{2} \int_{\tilde{r}_{\min }}^{\tilde{r}_{\max }} \frac{c^{-2} \alpha^{2} r^{-2}-2 m U_{\beta}(r)}{\left[2 m\left(-|\tilde{E}|+|\tilde{\alpha}| r^{-1}\right)-M^{2} r^{-2}\right]^{1 / 2}} d r ;
\end{aligned}
$$

$\tilde{r}_{\min } / \max$ are the roots of the radical in (4.2). The justification to replace $r_{\min } / \max$ by $\tilde{r}_{\min / \max }$ is given in the Remark following (4.4). The first integral in (4.2) is the action along the Kepler ellipse with major semiaxis $\tilde{a}=|\tilde{\alpha}| /(2|\tilde{E}|)$ and eccentricity $\tilde{e}=\sqrt{1-2|\tilde{E}| M^{2} /\left(|\tilde{\alpha}|^{2} m\right)}$, and $\tilde{r}_{\min / \max }=\tilde{a}(1 \mp \tilde{e})$. The action (4.2) is readily calculated as

$$
S=\pi\left(|\tilde{\alpha}| \sqrt{\frac{m}{2|\tilde{E}|}}-M\right)+\frac{\pi}{2} \frac{\tilde{\alpha}^{2}}{c^{2} M}-\pi \beta \sqrt{\frac{m}{2|\tilde{E}|}} J_{0}(\kappa \tilde{a} \tilde{e})(\cos (\kappa \tilde{a})+\lambda \sin (\kappa \tilde{a})),
$$

with $\kappa=m_{t} c / \hbar$ as defined in $(2.1)$. We may replace $(\tilde{E}, \tilde{\alpha})$ by $(E, \alpha)$ in the second and third term, as well as in the formulas for major semiaxis and eccentricity, because we consider only terms linear in $1 / c^{2}$ and $\beta$. In the first term we substitute

$$
|\tilde{\alpha}| \sqrt{\frac{m}{2|\tilde{E}|}}=|\alpha| \sqrt{\frac{m}{2|E|}}-\frac{3}{4} \frac{|\alpha|}{c^{2}} \sqrt{\frac{|E|}{2 m}}+O\left(\frac{1}{c^{4}}\right) .
$$

Remark. A short comment on the expansion procedures used here and in Sec. 2 . The integral (4.1) is of the type

$$
I(\epsilon)=\int_{A(\epsilon)}^{B(\epsilon)} F(\epsilon g(r), r) d r
$$


$A(\epsilon)$ and $B(\epsilon)$ are zeros of $F(\epsilon g(r), r)$. [In (4.1), $\beta$ and $1 / c^{2}$ correspond to $\epsilon$, of course.] We assume $A(\epsilon)=A(0)+\alpha \epsilon+\cdots$ and $B(\epsilon)=B(0)+\beta \epsilon+\cdots$, as well as the necessary smoothness of $F(x, r)$ and $g(r)$. We write (4.5) as

$$
\begin{aligned}
I(\epsilon) & =C(\epsilon) \int_{A(0)}^{B(0)} F(\epsilon g(y), y) d r, \\
y & =C(\epsilon)(r-A(0))+A(\epsilon), \\
C(\epsilon) & :=(B(\epsilon)-A(\epsilon)) /(B(0)-A(0)),
\end{aligned}
$$

and expand in $\epsilon ; C(\epsilon)=1+\gamma \epsilon+\cdots$,

$$
\begin{aligned}
C(\epsilon) F(\epsilon g(y), y)= & F(0, r)+\frac{\partial F(x=0, r)}{\partial x} g(r) \epsilon \\
& +\left(\gamma \frac{d(F(0, r) r)}{d r}+(\alpha-A(0) \gamma) \frac{d F(0, r)}{d r}\right) \epsilon+O\left(\epsilon^{2}\right) .
\end{aligned}
$$

The $r$ derivatives drop out in the integration (4.6), since $A(0)$ and $B(0)$ are zeros of $F(0, r)$. Therefore, to calculate $I(\epsilon)$ in first order in $\epsilon$, we may replace the integrand $F(\epsilon g(r), r)$ in (4.5) by the first two terms of the expansion (4.7), and the integration boundaries in (4.5) by the zeros of $F(0, r)$. A similar reasoning holds for the scattering integrals considered in Sec. 2 .

Bohr's quantization rules $^{24}$ read as

$$
S\left(r_{\min }, r_{\max }\right)=\pi \hbar n_{r}, \quad M=\hbar n_{\varphi},
$$

with radial and azimutal quantum numbers $n_{r}=0,1,2, \ldots$, and $n_{\varphi}=1,2, \ldots$, respectively. Solving (4.8) [with (4.3) and (4.4) inserted] for $E$, we obtain

$$
\begin{aligned}
& E\left(n, n_{\varphi}\right)=E_{n}\left[1+c^{-2} h\left(n, n_{\varphi}\right)+\beta g\left(n, n_{\varphi}\right)+O\left(\beta^{2}, c^{-4}, \beta c^{-2}\right)\right], \\
& h\left(n, n_{\varphi}\right)=\frac{\alpha^{2}}{\hbar^{2} n}\left(\frac{1}{n_{\varphi}}-\frac{3}{4} \frac{1}{n}\right), \\
& g\left(n, n_{\varphi}\right)=-\frac{2}{|\alpha|} J_{0}\left(\kappa a_{n} e\right)\left(\cos \left(\kappa a_{n}\right)+\lambda \sin \left(\kappa a_{n}\right)\right),
\end{aligned}
$$

with $n=n_{r}+n_{\varphi}, E_{n}=-\alpha^{2} m /\left(2 \hbar^{2} n^{2}\right), a_{n}=\hbar^{2} n^{2} /(|\alpha| m)$, and $e=\sqrt{1-n_{\varphi}^{2} / n^{2}}$. The term $c^{-2} h\left(n, n_{\varphi}\right)$, accounting for the relativistic fine structure splitting in lowest order, coincides with that obtained from the Dirac equation. ${ }^{28}$ (This coincidence is in fact exact, holding for all orders of the $1 / c^{2}$ expansion.)

In the following we give estimates for the tachyon mass and the coupling constant. At first we specify the boundary condition for the potential, by requiring a finite self-energy [see the discussion after (1.4)], which means to drop the cosine in (4.9) and to put $\lambda=1$. We start with the hydrogen atom, and introduce dimensionless constants $\hat{\alpha}, \hat{\beta}$, and $\hat{\kappa}$, via

$$
-\alpha=: \hbar c \hat{\alpha}, \quad \beta=: \hbar c \hat{\beta}, \quad \kappa=: \frac{m c}{\hbar} \hat{\alpha} \hat{\kappa} .
$$


$\hat{\beta}$ evidently is the tachyonic analogue to the electric fine structure constant $\hat{\alpha} \approx$ $1 / 137 . \hat{\kappa}$ denotes the tachyon mass in units of $m \hat{\alpha}$, where $m$ is the reduced mass of electron and proton. In (4.9) we substitute $\kappa a_{n}=n^{2} \hat{\kappa}$, and write the azimutal quantum number in standard quantum mechanical notation as $n_{\varphi}=j+1 / 2$. Wave numbers are denoted by $\tilde{\nu}_{n, j}=E_{n, j} /(h c)$, and energy levels are defined positive for convenience, so that $E_{n, j}=-E\left(n, n_{\varphi}\right)$.

We may now write, $\mathrm{cf}$. (4.9),

$$
\begin{aligned}
\frac{h}{m c} \tilde{\nu}_{n, j, l} & =\frac{1}{2} \frac{\hat{\alpha}^{2}}{n^{2}}+\frac{1}{2} \frac{\hat{\alpha}^{4}}{n^{4}}\left(\frac{n}{j+1 / 2}-\frac{3}{4}\right)+\frac{h}{m c} \tilde{\nu}_{n, j, l}^{\mathrm{QED}}+\frac{h}{m c} \tilde{\nu}_{n, j}^{\mathrm{tach}}, \\
\frac{h}{m c} \tilde{\nu}_{n, j}^{\mathrm{tach}} & :=-\frac{\hat{\alpha} \hat{\beta}}{n^{2}} \sin \left(\hat{\kappa} n^{2}\right) J_{0}\left(\hat{\kappa} n^{2} \sqrt{1-\frac{(j+1 / 2)^{2}}{n^{2}}}\right),
\end{aligned}
$$

where we have indicated by $\tilde{\nu}_{n, j, l}^{\mathrm{QED}}$ the correction stemming from higher order terms of the relativistic fine splitting and the Lamb shift, labeled by the usual quantum numbers. ${ }^{28,29}$ The procedure will be to substitute on the left side of Eq. (4.11) the experimental wave number, and for the first three terms on the right side the theoretical result based on Lamb shift calculations. In this way equations for the tachyon mass $\hat{\kappa}$ and the coupling constant $\hat{\beta}$ can be obtained.

Remark. Since $J_{0}(z \rightarrow \infty) \sim \sqrt{2 /(\pi z)} \cos (z-\pi / 4)$, the contribution of the tachyon potential to the wave number decreases with $\hat{\kappa}^{-1 / 2}$ if the tachyon mass is large. There is one exception, namely Yrast states, $n=j+1 / 2$, because in this case the argument of the Bessel function is zero. If the orbit is elliptical, the variation of the orbital radius is about $2 e a_{n}$ (the difference between aphel and perihel) with eccentricity and Bohr radius as defined after (4.9). The number of sign changes of the tachyon potential along this distance is approximately $2 e n^{2} \hat{\kappa} / \pi$. Thus, for $\hat{\kappa} \rightarrow \infty$, there is an increasing number of sign changes of the tachyon potential along an elliptical orbit, so that the average value of the tachyon potential along the orbit approaches zero in this limit. But in the case of Yrast states, the orbits are circular, therefore the contribution of the tachyon potential to the energy levels does not diminish with increasing mass, as there is no sign change along the orbit. However, that is also likely to change in a wave mechanical treatment, as orbital radii can then only be defined as expectation values. Therefore, also for Yrast states, the contribution of the tachyon potential will diminish with increasing mass, due to sign changes of the potential in the spherical shell in which the bulk of the probability density is located. As a semiclassical treatment is likely to be insufficient if the tachyon mass is large, we focus in this paper on a lower bound for it. But even a potential with a large tachyon mass may well be observable, because this averaging effect can be compensated by a large coupling constant.

We start with the $1 S-2 S_{1 / 2}$ transition in hydrogen, and write the difference of experimental and theoretical frequencies in terms of measured and theoretical Lamb shifts, $\nu_{1 S-2 S}^{\mathrm{ex}}-\nu_{1 S-2 S}^{\mathrm{th}}=L_{1 S}^{\mathrm{th}}-L_{1 S}^{\mathrm{ex}}+L_{2 S}^{\mathrm{ex}}-L_{2 S}^{\mathrm{th}}$. We take for $L_{1 S}^{\mathrm{ex}}$ the average of $8172.798 \mathrm{MHz}, 8172.827 \mathrm{MHz}, 8172.874 \mathrm{MHz}$, and $8172.876 \mathrm{MHz}$ 
[cf. Refs. 30-33, respectively], and use $L_{1 S}^{\mathrm{th}}=8172.802 \mathrm{MHz}$, cf. Refs. 29 and 33. The difference of the $2 \mathrm{~S}$ Lamb shifts is negligible in view of the very large error bounds on $L_{1 S}^{\mathrm{ex}, \text { th }}$, see the papers cited above, and so we find $\nu_{1 S-2 S}^{\mathrm{ex}}-\nu_{1 S-2 S}^{\mathrm{th}} \approx-0.042 \mathrm{MHz}$. The theoretical frequency corresponds to the first three terms in (4.11), so that we arrive at

$$
0.042 \mathrm{MHz} \frac{h}{m c^{2}}=\hat{\alpha} \hat{\beta} \sin \hat{\kappa}-\frac{\hat{\alpha} \hat{\beta}}{4} J_{0}(\hat{\kappa} 2 \sqrt{3}) \sin (4 \hat{\kappa}) .
$$

Next we turn to the $2 S_{1 / 2}-2 P_{3 / 2}$ interval. We have an experimental value of 9911.200 MHz, [cf. Ref. 34], and a theoretical one of $9911.2025 \mathrm{MHz}$, [cf. Refs. 29 and 35]. We thus obtain, by means of (4.11) and (4.12),

$$
0.0025 \mathrm{MHz} \frac{h}{m c^{2}}=\frac{\hat{\alpha} \hat{\beta}}{4} \sin (4 \hat{\kappa})\left(J_{0}(\hat{\kappa} 2 \sqrt{3})-1\right) .
$$

Combining (4.13) and (4.14), we find

$$
4.2 \sin (4 \hat{\kappa})\left(J_{0}(\hat{\kappa} 2 \sqrt{3})-1\right)=\sin \hat{\kappa}-(1 / 4) \sin (4 \hat{\kappa}) J_{0}(\hat{\kappa} 2 \sqrt{3}) .
$$

The smallest solution of this equation is $\hat{\kappa} \approx 0.82$. This yields the estimate $3.06 \mathrm{keV} / c^{2}$ for the lowest possible tachyon mass $m \hat{\alpha} \hat{\kappa}$, cf. (4.10). With $J_{0}(2.84) \approx$ -0.20 , and $h /\left(m c^{2}\right) \approx 8.1 \times 10^{-21} \mathrm{~s}$, we obtain, via (4.13), the tachyonic fine structure constant $\hat{\beta} \approx 6.4 \times 10^{-14}$.

Remarks. (1) Equation (4.15) admits a discrete set of possible solutions, because it gets periodic for large $\hat{\kappa}$, but one has to keep in mind that for large tachyon mass the Bohr-Sommerfeld quantization breaks down. (2) The confidence intervals of the measured and theoretical frequencies used in deriving (4.15) overlap; so there is actually no established discrepancy between the theoretical and experimental results, at least if one assumes a suitable proton radius. ${ }^{33}$ The purpose of this investigation is not so much to improve on the theoretical Lamb shift, but rather to show how one can accommodate the tachyon potential in atomic spectra, and in doing so, to obtain estimates of the tachyon mass and the coupling constant. On the other hand, although the coefficient 4.2 in Eq. (4.15) is uncertain (because of the large error bounds on the theoretical and experimental $1 \mathrm{~S}$ Lamb shift, ${ }^{33}$ and the large experimental confidence interval for the $2 S_{1 / 2}-2 P_{3 / 2} \operatorname{transition}^{34}$ ), this does not substantially affect the smallest solution; in the range $4.2_{-3.6}^{+6.2}$, we find $\hat{\kappa}=0.82_{+0.3}^{-0.02}$. We do not attempt to give an error bound on $\hat{\beta} \approx 6.4 \times 10^{-14}$, but rather check the quality of this result in hydrogenic ions.

We study hydrogenlike ions of medium and high $Z$. In (4.10), this requires the following substitutions:

$$
\hat{\alpha} \rightarrow \hat{\alpha} Z, \quad \hat{\beta} \rightarrow \hat{\beta} Z^{7}, \quad \hat{\kappa} \rightarrow \frac{1-m_{e} / m_{\mathrm{nuc}}}{1-m_{e} / m_{p}} \frac{1}{Z} \hat{\kappa} ;
$$

$m$ in (4.10) now denotes the reduced mass of electron and nucleus. The $Z$-scaling of $\hat{\alpha}$ is evident, and there is strong experimental evidence for the $Z^{7}$-scaling of $\hat{\beta}$, presented below. The tachyon mass is independent of the charge and the mass of the 
source generating the potential, which results in the indicated scaling of $\hat{\kappa}$. In the following we neglect the mass ratios in (4.16) stemming from the reduced masses, and use $m=0.51 \mathrm{MeV} / c^{2}, \hat{\kappa}=0.82$, and $\hat{\beta}=6.4 \times 10^{-14}$.

At first we consider the $2 s_{1 / 2}-2 p_{3 / 2}$ transition in hydrogenlike sulfur, $\mathrm{S}^{15+}$. This is a rare case where theoretical and experimental error bounds do not overlap. The discrepancy between experiment ${ }^{36}$ and theoretical standard result ${ }^{37,38}$ is $\Delta E^{\text {ex-th }}:=$ $E_{2 S-2 P}^{\mathrm{ex}}-E_{2 S-2 P}^{\mathrm{th}} \approx-4.5(3.0) \times 10^{-4} \mathrm{eV}$. The energy shift induced by the tachyon potential reads as $E_{n, j}^{\text {tach }}:=h c \tilde{\nu}_{n, j}^{\text {tach }}$, with $(\hat{\alpha}, \hat{\beta}, \hat{\kappa}) \rightarrow\left(\hat{\alpha} Z, \hat{\beta} Z^{7}, \hat{\kappa} / Z\right)$ substituted in (4.12). This amounts to

$$
\frac{E_{2 S-2 P}^{\mathrm{tach}}}{m c^{2}}=\frac{\hat{\alpha} \hat{\beta} Z^{8}}{4} \sin \left(4 \hat{\kappa} Z^{-1}\right)\left(J_{0}\left(\hat{\kappa} Z^{-1} 2 \sqrt{3}\right)-1\right) \approx-3 \hat{\alpha} \hat{\beta} \hat{\kappa}^{3} Z^{5}
$$

where we used $J_{0}(z)=1-z^{2} / 4+O\left(z^{4}\right)$. With $Z=16$, we find $E_{2 S-2 P}^{\mathrm{tach}} /\left(m c^{2}\right) \approx$ $-8.1 \times 10^{-10}$, which is to be compared to $\Delta E^{\text {ex-th }} /\left(m c^{2}\right) \approx-8.8 \times 10^{-10}$. This is excellent agreement, the tachyonic correction $E_{2 S-2 P}^{\text {tach }}$ almost completely accounts for the discrepancy of measured and theoretical Lamb shift.

Next we consider the Ly- $\alpha_{1}$ line, $1 s_{1 / 2}-2 p_{3 / 2}$, in hydrogenic argon, $\mathrm{Ar}^{17+}$. We have $E_{1 S-2 P}^{\mathrm{th}}=3.3230191 \mathrm{keV}$ and $E_{1 S-2 P}^{\mathrm{ex}}=3.323016(17) \mathrm{keV}$, [cf. Refs. 37-39] so that $\Delta E^{\text {ex-th }} /\left(m c^{2}\right):=\left(E_{1 S-2 P}^{\mathrm{ex}}-E_{1 S-2 P}^{\mathrm{th}}\right) /\left(m c^{2}\right) \approx-6 \times 10^{-9}$. We find, quite analogously to $(4.17)$,

$$
\frac{E_{1 S-2 P}^{\mathrm{tach}}}{m c^{2}}=-\hat{\alpha} \hat{\beta} Z^{8}\left(\sin \left(\hat{\kappa} Z^{-1}\right)-\frac{1}{4} \sin \left(4 \hat{\kappa} Z^{-1}\right)\right) \approx-\frac{5}{2} \hat{\alpha} \hat{\beta} \hat{\kappa}^{3} Z^{5},
$$

and we arrive at $E_{1 S-2 P}^{\text {tach }} /\left(m c^{2}\right) \approx-1.2 \times 10^{-9}(Z=18)$. Based on (4.18), we can predict a transition energy $E_{1 S-2 P}=E^{\text {th }}+E^{\text {tach }}=3.3230185 \mathrm{keV}$, which is well within the confidence interval of the quoted experimental result.

As for the Ly- $\alpha_{2}$ transition in $\mathrm{Ar}^{17+}, 1 s_{1 / 2}-2 p_{1 / 2}$, we have $E_{1 S-2 P}^{\mathrm{th}}=$ $3.3182033 \mathrm{keV}$ and $E_{1 S-2 P}^{\mathrm{ex}}=3.318198(17) \mathrm{keV}$, cf. Refs. $37-39$, so that $\Delta E^{\mathrm{ex}-\mathrm{th}} /$ $\left(m c^{2}\right) \approx-1.0 \times 10^{-8}$. We now obtain, analogous to $(4.18)$,

$$
\frac{E_{1 S-2 P}^{\mathrm{tach}}}{m c^{2}}=-\hat{\alpha} \hat{\beta} Z^{8}\left(\sin \left(\hat{\kappa} Z^{-1}\right)-\frac{1}{4} \sin \left(4 \hat{\kappa} Z^{-1}\right) J_{0}\left(\hat{\kappa} Z^{-1} 2 \sqrt{3}\right)\right) \approx-\frac{11}{2} \hat{\alpha} \hat{\beta} \hat{\kappa}^{3} Z^{5},
$$

or $E_{1 S-2 P}^{\text {tach }} /\left(m c^{2}\right) \approx-2.7 \times 10^{-9}$. Our prediction for the transition energy is thus $E_{1 S-2 P}=E^{\text {th }}+E^{\text {tach }}=3.3182019 \mathrm{keV}$, very close to $E_{1 S-2 P}^{\text {ex }}$.

For the Ly- $\alpha_{1}$ line of hydrogenlike krypton, $\mathrm{Kr}^{35+}, E_{1 S-2 P}^{\mathrm{th}}=13.509046 \mathrm{keV}$ and $E_{1 S-2 P}^{\mathrm{ex}}=13.50895(50) \mathrm{keV}$, [cf. Refs. 37, 38 and 40$]$ so that $\Delta E^{\text {ex-th }} /\left(m c^{2}\right) \approx$ $-1.9 \times 10^{-7}$. We arrive, via $(4.18)(Z=36)$, at $E_{1 S-2 P}^{\text {tach }} /\left(m c^{2}\right) \approx-3.9 \times 10^{-8}$, and our prediction is $E_{1 S-2 P}=13.509026 \mathrm{keV}$, again well within the error bound of $E_{1 S-2 P}^{\mathrm{ex}}$.

For the Ly- $\alpha_{1}$ line of $\mathrm{Ni}^{27+}$, we have $\Delta E^{\text {ex-th }} /\left(m c^{2}\right) \approx 5.1 \times 10^{-8}$; the measured $1 \mathrm{~S}$ Lamb shift reads $L_{1 S}^{\mathrm{ex}}=5.07(10) \mathrm{eV}$, and the theoretical result is $L_{1 S}^{\mathrm{th}}=5.096 \mathrm{eV}$, 
[cf. Refs. 37, 38 and 41]. $\left(\Delta E^{\mathrm{ex}-\mathrm{th}}=L_{1 S}^{\mathrm{th}}-L_{1 S}^{\mathrm{ex}}+L_{2 P}^{\mathrm{ex}}-L_{2 P}^{\mathrm{th}}\right.$, and the difference of the experimental and theoretical $2 P_{3 / 2}$ shifts is assumed to be negligible compared to the difference of the much larger $1 \mathrm{~S}$ shifts.) We find, by means of (4.18) (with $Z=28), E_{1 S-2 P}^{\text {tach }} /\left(m c^{2}\right) \approx-1.1 \times 10^{-8}$, which leads to a predicted Lamb shift of $L_{1 S} \approx L_{1 S}^{\mathrm{th}}-E_{1 S-2 P}^{\mathrm{tach}}=5.102 \mathrm{eV}$, well within the confidence interval of $L_{1 S}^{\mathrm{ex}}$.

For hydrogenlike gold, $\mathrm{Au}^{78+}$, the ground-state Lamb shift reads as $L_{1 S}^{\mathrm{th}}=$ $205.7 \mathrm{eV}$, and $L_{1 S}^{\mathrm{ex}}=202.3(8.0) \mathrm{eV}$, cf. Refs. 42-44. As in the case of nickel, we consider the Ly- $\alpha_{1}$ transition, and neglect the difference of the small $2 P_{3 / 2}$ shifts, so that $\Delta E^{\mathrm{ex}-\mathrm{th}} \approx L_{1 S}^{\mathrm{th}}-L_{1 S}^{\mathrm{ex}}$, which yields $\Delta E^{\mathrm{ex}-\mathrm{th}} /\left(m c^{2}\right) \approx 6.7 \times 10^{-6}$. With $(4.18)$ and $Z=79$, we find $E_{1 S-2 P}^{\text {tach }} /\left(m c^{2}\right) \approx-2.0 \times 10^{-6}$. This leads to the prediction $L_{1 S} \approx L_{1 S}^{\mathrm{th}}-E_{1 S-2 P}^{\mathrm{tach}}=206.7 \mathrm{eV}$, still within the confidence interval of $L_{1 S}^{\mathrm{ex}}$, though only narrowly.

Finally, for $U^{91+}, L_{1 S}^{\mathrm{th}}=465.6 \mathrm{eV}$ and $L_{1 S}^{\mathrm{ex}}=469(13) \mathrm{eV}$, [cf. Refs. 42-44], so that $\Delta E^{\text {ex-th }} /\left(m c^{2}\right) \approx-6.7 \times 10^{-6}$, and the contribution of the tachyon potential to the Ly- $\alpha_{1}$ interval is $E_{1 S-2 P}^{\text {tach }} /\left(m c^{2}\right) \approx-4.2 \times 10^{-6}$ (via (4.18), with $Z=92$ ), yielding a predicted Lamb shift of $L_{1 S}=467.7 \mathrm{eV}$, very close to $L_{1 S}^{\mathrm{ex}}$.

These results are strong evidence for a $Z^{7}$-scaling of the tachyonic coupling constant, assumed in (4.16). Returning to the potential (2.1) [with $\lambda=1$ and the cosine dropped], we may now write

$$
\alpha=\frac{e e^{\prime}}{4 \pi}=\hbar c \hat{\alpha} Z Z^{\prime}, \quad \beta=-\frac{q q^{\prime}}{4 \pi}=-\hbar c \hat{\beta}\left(Z Z^{\prime}\right)^{7}, \quad \kappa=\frac{m c}{\hbar} \hat{\alpha} \hat{\kappa} .
$$

$Z$ is here a positive or negative integer defining electric and tachyonic charge, $e=$ $\sqrt{4 \pi \hbar c \hat{\alpha}} Z$ and $q=\sqrt{4 \pi \hbar c \hat{\beta}} Z^{7}$, so that $q / e=\sqrt{\hat{\beta} / \hat{\alpha}} Z^{6}$. (To summarize, $\hat{\alpha} \approx 1 / 137$, $\hat{\beta} \approx 6.4 \times 10^{-14}, m \approx 0.51 \mathrm{MeV} / c^{2}, \hat{\kappa} \approx 0.82$, and $m_{t}=m \hat{\alpha} \hat{\kappa} \approx 3.06 \mathrm{keV}$.) The tachyon-electron mass ratio reads as $m_{t} / m \approx 6.0 \times 10^{-3}$. We readily derive

$$
\frac{q q^{\prime}}{e e^{\prime}} \approx \frac{\left(Z Z^{\prime}\right)^{6}}{146}\left(\frac{m_{t}}{m}\right)^{4}
$$

the coefficient $1 / 146$ in this formula is based on the numerical values of $\hat{\kappa}$ and $\hat{\beta}$. As pointed out in the Remarks following (4.15), the value of $\hat{\beta}$ is uncertain, and it is tempting to replace $1 / 146$ by the fine structure constant $\hat{\alpha}$, and then to regard (4.21) as an exact relation equivalent to

$$
\hat{\beta}=\hat{\alpha}^{6} \hat{\kappa}^{4}
$$

The numerical value of $\hat{\kappa}$ remains unchanged, and $\hat{\beta} \approx 6.8 \times 10^{-14}$. It is easy to check, that this mildly modified value of the tachyonic fine structure constant does not affect the above predictions for the transition energies and Lamb shifts in any significant way (a change of \pm 1 in the last decimal digit). Relation (4.22) is of course only conjectured on numerical grounds. It means that this field theory for tachyons involves only one new fundamental constant, the tachyon mass. 


\section{Conclusion}

If one contemplates on the possibility of superluminal motion, one is faced with the choice of an interaction mechanism with subluminal matter. A strictly causal theory of geodesically moving superluminal signals is straightforward to obtain in a cosmological setting, ${ }^{9,11}$ but it is less obvious to define realistic interactions of tachyons with matter, i.e. to attach a physical meaning to the term "superluminal signal." Quantization attempts ${ }^{1,3}$ of scalar field theories with superluminal modes have not led to a consistent theory, and quantum tachyons still remain to be properly defined. So it seems advisable to search for tachyons on a classical or semiclassical basis first. In this paper, a classical field theory of superluminal wave propagation is investigated, a real Proca field with negative mass square, which admits tachyonic modes. We derived the potential of a static point source, and we studied how it affects scattering and bound states if added to the Coulomb potential as a perturbation. We calculated the classical cross-section in various limits, cf. Sec. 2, and found that the tachyon potential substantially modifies the Coulomb cross-section in the limit of large impact parameter, where rainbow scattering applies, cf. Sec. 3.

In Sec. 4, we derived a lower bound on the tachyon mass as well as an estimate for the coupling constant of the tachyon potential, and related tachyonic to electric charge. All that was done on the assumption that the tachyon potential, added as a perturbation to the Coulomb potential, cf. (2.1), can account for the discrepancy of experimental and theoretical Lamb shifts in hydrogenic ions, and quantitative evidence was pointed out to support that. In this way we got a first, though indirect, clue for the existence of superluminal signal transfer, by virtue of the static potential of this superluminal field theory. There is also a tachyonic counterpart to the magnetic moment of the nucleus, inducing level shifts in the hyperfine structure. This could provide another feasible and quite independent test of this field theory, based on the ground state hyperfine splitting of hydrogen and hydrogenlike ions. Throughout this paper we used classical and semiclassical methods. Given the small coupling constant of the tachyon potential, a wave mechanical calculation of the level shifts induced by the tachyon potential is certainly not out of reach. This is all the more important as Bohr-Sommerfeld quantization is expected to break down in the limit of large tachyon mass, cf. the Remark following (4.12).

One can consider atoms in equilibrium with a mixture of photon and tachyon black-body radiation, and study the ratio of photonic and tachyonic transition rates. The derivation of tachyonic transition rates is quite analogous to photonic ones, by standard equilibrium arguments ${ }^{45}$ not repeated here, but we sketch how this ratio relates to the Einstein coefficients and the photonic and tachyonic spectral energy densities and give some estimates.

The quotient of the dipole matrix elements of tachyonic and electric charge and the absorption coefficients read

$$
\frac{\left|D_{m n}^{q}\right|^{2}}{\left|D_{m n}^{e}\right|^{2}}=\frac{\hat{\beta}}{\hat{\alpha}}, \quad B_{m n}^{e, q}=\frac{16}{3} \frac{\pi^{4}}{h^{2}}\left|D_{m n}^{e, q}\right|^{2},
$$


and the coefficients for spontaneous emission connect to the $B$ coefficients as

$$
A_{m n}^{e}=4 \frac{h \nu^{3}}{c^{3}} B_{m n}^{e}, \quad A_{m n}^{q}=4 \frac{h \nu^{2}}{c^{3}} \sqrt{\nu^{2}+\frac{m_{t}^{2} c^{4}}{h^{2}}} B_{m n}^{q} .
$$

The spectral energy densities of tachyon and photon black-body radiation relate as ${ }^{19}$

$$
\rho_{\mathrm{tach}}(\nu)=\frac{\sqrt{\nu^{2}+m_{t}^{2} c^{4} / h^{2}}}{\nu} \rho_{\mathrm{ph}}(\nu) .
$$

The ratio of tachyon and photon transition rates is the same for absorptive, stimulated, and spontaneous transitions,

$$
\frac{R_{m n}^{\mathrm{tach}}}{R_{m n}^{\mathrm{ph}}}:=\frac{B_{m n}^{q}}{B_{m n}^{e}} \frac{\rho_{\mathrm{tach}}(\nu)}{\rho_{\mathrm{ph}}(\nu)}=\frac{A_{m n}^{q}}{A_{m n}^{e}} \sim \frac{\hat{\beta}}{\hat{\alpha}} \frac{m_{t} c^{2}}{h \nu} ;
$$

the asymptotic relation holds for $m_{t} c^{2} /(h \nu) \gg 1$. Using $m_{t} \approx 3.06 \mathrm{keV} / c^{2}$ and $\hat{\beta} / \hat{\alpha} \approx 9.3 \times 10^{-12}$, cf. the end of Sec. 4 , we find $R^{\text {tach }} / R^{\mathrm{ph}} \approx 2.8 \times 10^{-9}$ for the Lyman- $\alpha$ lines of hydrogen $(10.2 \mathrm{eV})$. The $2 s_{1 / 2}-2 p_{1 / 2}$ transition in hydrogen $\left(4.4 \times 10^{-6} \mathrm{eV}\right)$ is much more likely to be effected by tachyons, since $R^{\mathrm{tach}} / R^{\mathrm{ph}} \approx$ $6.5 \times 10^{-3}$.

Finally we turn to the cosmic tachyon background radiation. ${ }^{19}$ One readily calculates the ratio of photon and tachyon density as well as the ratio of the corresponding energy densities in the low temperature regime, $m_{t} c^{2} /(k T) \gg 1$,

$$
\frac{N_{\mathrm{tach}}}{N_{\mathrm{ph}}} \sim \frac{\pi^{2}}{12 \zeta(3)} \frac{m_{t} c^{2}}{k T}, \quad \frac{U_{\mathrm{tach}}}{U_{\mathrm{ph}}} \sim \frac{30 \zeta(3)}{\pi^{4}} \frac{m_{t} c^{2}}{k T} .
$$

We find, with a background temperature of $2.73 \mathrm{~K}, N_{\mathrm{tach}} / N_{\mathrm{ph}} \approx 8.9 \times 10^{6}$ and $U_{\text {tach }} / U_{\mathrm{ph}} \approx 4.8 \times 10^{6}$. As pointed out above, the high tachyon density can only in part compensate for the small ratio of tachyonic and electric fine structure constants, which makes the tachyon background difficult to observe. On the other hand, $3 \mathrm{keV}$ is only a lower bound on the tachyon mass, a larger mass increases both the tachyon density and the tachyonic fine structure constant, cf. (4.22) and the Remark following (4.12), and thus the absorption rate for tachyon radiation.

A final comment on superluminal signals, which we have not addressed at all in this paper. Superluminal signal transfer is effected by wave solutions of the field equations (1.2), cf. Ref. 20. As the field is conformally coupled to the background metric, the semiclassical approximation is exact, which means that the classical tachyonic action $S$ can be identified with the phase of the spectral elementary modes, so that the integration constants in $S$ appear as spectral parameters. [The tachyonic action is of course defined by a Hamilton-Jacobi equation with negative mass square, $g^{\mu \nu} S_{, \mu} S_{, \nu}=\mu^{2}$, with $\mu^{2}$ and $g_{\mu \nu}$ as in (1.2). This equation for geodesically moving tachyons should not be confused with the Hamilton-Jacobi equation for subluminal particles in the tachyon potential, cf. (2.2).] Hence, the frequencies of 
the spectral waves relate to the velocity/energy of the classical tachyonic world-lines exactly via the Einstein/de Broglie relation, ${ }^{3,7}$

$$
h \nu=m_{t} c^{2}\left(\frac{\mathbf{v}^{2}}{c^{2}}-1\right)^{-1 / 2} .
$$

Tachyons do not carry any kind of charge. Tachyonic world-lines are analogous to the light-rays of geometric optics, and, by virtue of (1.2) and (5.6), tachyons can be viewed as a kind of photons with negative mass square. They are transversally propagating spin one particles, cf. Ref. 20, where the spectral resolution of (1.2) is discussed in detail. The tachyon potential in (2.1) is just a static solution of the field equations, analogous to the Coulomb potential. Electric and tachyonic charge is carried by massive subluminal particles, the sources of tachyonic and electromagnetic fields.

Equation (5.6) determines the speed of tachyons emitted in atomic transitions. If a tachyon is generated by a Lyman- $\alpha$ transition in a heavy hydrogenic ion, $\mathrm{cf}$. Sec. 4, then its velocity is rather close to the speed of light. But the opposite asymptotic limit, $|\mathbf{v}| \sim 3.06 \mathrm{keV} c /(h \nu)$, applies to the $2 s_{1 / 2}-2 p_{1 / 2}$ Lamb shift splitting in hydrogen, yielding $|\mathbf{v}| \sim 6.8 \times 10^{8} c \approx 0.21 \mathrm{Gpc} / \mathrm{yr}$, a comfortable speed of signal transfer on the distance scale of nearby galaxies.

\section{Acknowledgments}

The author acknowledges the support of the Japan Society for the Promotion of Science. Initial stages of this work were carried out in the Indian Institute of Astrophysics, Bangalore. I would like to thank Prof. C. V. Vishveshwara for initiating my visit to the IIAP. An inspiring stay at the Tata Institute of Fundamental Research, Bombay, is likewise gratefully acknowledged.

\section{References}

1. S. Tanaka, Prog. Theor. Phys. 24, 171 (1960).

2. Ya. P. Terletsky, Sov. Phys. Dokl. 5, 782 (1961).

3. G. Feinberg, Phys. Rev. 159, 1089 (1967).

4. L. Parker, Phys. Rev. 188, 2287 (1969).

5. R. Newton, Science 167, 1569 (1970).

6. A. D. Dolgov and I. D. Novikov, Phys. Lett. B442, 82 (1998).

7. G. Feinberg, Sci. Am. 222 (2), 69 (1970).

8. A. Sommerfeld, Proc. Konink. Akad. Wet. (Amsterdam) Sec. Sci. 7, 346 (1904).

9. R. Tomaschitz, Chaos, Solitons \& Fractals 7, 753 (1996); ibid. 8, 761 (1997).

10. R. Tomaschitz, Chaos, Solitons \& Fractals 9, 1199 (1998).

11. R. Tomaschitz, Int. J. Mod. Phys. D7, 279 (1998).

12. G. Wentzel, Quantum Theory of Fields (Interscience, New York, 1949).

13. A. S. Goldhaber and M. M. Nieto, Rev. Mod. Phys. 43, 277 (1971).

14. E. A. Milne, Kinematic Relativity (Clarendon, Oxford, 1948).

15. P. A. M. Dirac, Nature 139, 323 (1937).

16. F. J. Dyson, in Aspects of Quantum Theory, eds. A. Salam and E. P. Wigner (Cambridge University Press, 1972). 
17. R. Tomaschitz, Int. J. Theor. Phys. 37, 1121 (1998).

18. R. Tomaschitz, Astrophys. \& Space Sci. 259, 255 (1998).

19. R. Tomaschitz, Int. J. Mod. Phys. A14, 4275 (1999).

20. R. Tomaschitz, Class. Quantum Grav. 16, 3349 (1999).

21. M. Born and L. Infeld, Proc. R. Soc. (London) A144, 425 (1934).

22. K. W. Ford and J. A. Wheeler, Ann. Phys. (N.Y.) 7, 259 (1959), ibid. 7, 287 (1959).

23. R. Newton, Scattering Theory of Waves and Particles (McGraw-Hill, New York, 1966).

24. L. D. Landau and E. M. Lifshitz, Quantum Mechanics: Nonrelativistic Theory, 3rd ed. (Pergamon, London, 1991).

25. E. Fischbach and C. Talmadge, Nature 356, 207 (1992).

26. G. N. Watson, A Treatise on the Theory of Bessel Functions (Cambridge University Press, Cambridge, 1966).

27. W. Magnus, F. Oberhettinger and R. P. Soni, Formulas and Theorems for the Special Functions of Mathematical Physics (Springer, Berlin, 1966).

28. V. B. Beresteckij, E. M. Lifshitz and L. P. Pitaevskii, Quantum Electrodynamics (Pergamon, Oxford, 1982).

29. K. Pachucki et al., J. Phys. B29, 177 (1996).

30. S. Bourzeix et al., Phys. Rev. Lett. 76, 384 (1996).

31. D. J. Berkeland, E. A. Hinds and M. G. Boshier, Phys. Rev. Lett. 75, 2470 (1995).

32. M. Weitz et al., Phys. Rev. A52, 2664 (1995).

33. Th. Udem et al., Phys. Rev. Lett. 79, 2646 (1997).

34. E. W. Hagley and F. M. Pipkin, Phys. Rev. Lett. 72, 1172 (1993).

35. U. Jentschura and K. Pachucki, Phys. Rev. A54, 1853 (1996).

36. A. P. Georgiadis et al., Phys. Lett. A115, 108 (1986).

37. W. R. Johnson and G. Soff, At. Data Nucl. Data Tables 33, 405 (1985).

38. R. D. Deslattes et al., in Atomic and Molecular Data and Their Applications, eds. P. J. Mohr and W. L. Wiese (AIP Conf. Proc. 434, 1998).

39. H. F. Beyer et al., J. Phys. B18, 207 (1985).

40. M. Tavernier et al., J. Phys. B18, L327 (1985).

41. H. F. Beyer et al., Phys. Rev. A43, 223 (1991).

42. T. Beier et al., Phys. Lett. A236, 329 (1997).

43. H. F. Beyer et al., Z. Phys. D35, 169 (1995).

44. H. F. Beyer and Th. Stöhlker, in Frontier Tests of QED and Physics of the Vacuum, eds. E. Zavattini et al. (Haron Press, Sofia, 1998).

45. S. Chandrasekhar, Radiative Transfer (Dover, New York, 1960). 